\title{
Inactivation of Cryptosporidium parvum Oocysts in Fresh Apple Cider by UV Irradiation
}

\author{
D. E. Hanes, ${ }^{1 *}$ R. W. Worobo, ${ }^{2}$ P. A. Orlandi, ${ }^{3}$ D. H. Burr, ${ }^{1}$ M. D. Miliotis,${ }^{3}$ M. G. Robl, ${ }^{1}$ \\ J. W. Bier, ${ }^{3}$ M. J. Arrowood, ${ }^{4}$ J. J. Churey, ${ }^{2}$ and G. J. Jackson ${ }^{3}$ \\ Center for Food Safety and Applied Nutrition, U.S. Food and Drug Administration, Laurel, Maryland ${ }^{1}$; U.S. Food and Drug \\ Administration, Washington, D.C. ${ }^{3}$; Cornell University Department of Food Science and Technology, New York \\ State Agricultural Experiment Station, Geneva, New York ${ }^{2}$; and Division of Parasitic Diseases, Centers for
} Disease Control and Prevention, Atlanta, Georgia ${ }^{4}$

Received 20 September 2001/Accepted 19 April 2002

\begin{abstract}
This study evaluated the efficacy of UV irradiation on the inactivation of Cryptosporidium parvum oocysts in fresh apple cider. Cider was inoculated with oocysts and exposed to $14.32 \mathrm{~mJ}$ of UV irradiation $/ \mathrm{cm}^{2}$. Oocyst viability was assessed with the gamma interferon gene knockout (GKO) mouse and infant BALB/cByJ mouse models. All GKO mice challenged with UV-treated cider demonstrated no morbidity or mortality, and infant $\mathrm{BALB} / \mathrm{c}$ mice challenged with treated cider were negative for the presence of $C$. parvum. In contrast, the GKO mice challenged with non-UV-treated inoculated cider died and the parasite was detected in the ileums of all challenged infant mice. This study shows that UV irradiation can be used to inactivate $C$. parvum in fresh apple cider.
\end{abstract}

Cryptosporidium parvum is a protozoan parasite that causes mild to severe gastroenteritis in humans and animals $(9,18)$. Disease in humans is most severe in the very young, the aged, and the immunocompromised, such as AIDS patients (6). The symptoms of cryptosporidiosis are normally mild and self-limiting in immunocompetent humans, but in immunocompromised patients, illness is often chronic and can be life threatening $(10,22)$. There is currently no consistently effective drug for the treatment of cryptosporidiosis; therefore, elimination of viable parasites in food and drink is highly desirable, especially for individuals in high-risk groups. C. parvum is transmitted primarily by the fecal-oral route, usually through exposure to infected animals or contact with humans infected with the parasite $(9,18)$. Outbreaks of cryptosporidiosis have also been associated with the consumption of fresh, unpasteurized cider made from apples most likely contaminated with cattle feces $(4,8,15,16)$. Cider-associated outbreaks of cryptosporidiosis have prompted the cider industry to search for a process that inactivates $C$. parvum oocysts without affecting the organoleptic qualities of fresh cider and while being cost-effective for small cider mills. UV irradiation is effective against a number of microorganisms that may be present in drinking water (3, 26), including $C$. parvum oocysts (5). UV irradiation has been effective in the reduction of Escherichia coli O157:H7 in unpasteurized apple cider (20, 25), and Harrington and Hills (13) demonstrated that cider exposed to UV for 54 showed no alterations in flavor quality when held at $22^{\circ} \mathrm{C}$ for up to 35 days. This study examined the effectiveness of using a CiderSure 3500A apparatus for UV irradiation to inactivate $C$. parvum oocysts in fresh apple cider. The results demonstrated that a greater than $5 \log _{10}$ reduction in oocyst viability can be ob-

\footnotetext{
* Corresponding author. Mailing address: Food and Drug Administration, 8301 Muirkirk Rd., Laurel, MD 20708. Phone and fax: (301) 827-8079. E-mail: dhanes@cfsan.fda.gov.
}

tained by exposing experimentally contaminated apple cider to $14.32 \mathrm{~mJ}$ of UV irradiation/ $/ \mathrm{cm}^{2}$ for 1.2 to $1.9 \mathrm{~s}$. The CiderSure apparatus used for these studies is small and portable and ought to prove cost-effective for both large and small cider operations.

Cryptosporidium parvum oocysts of the strain Iowa which were purified from the stools of experimentally infected Holstein bull calves $(1 \mathrm{a}, 2)$ were used for all studies. Purified oocysts were enumerated in a hemocytometer, adjusted to a final concentration of $1 \times 10^{8}$ to $10 \times 10^{8}$ oocysts $/ \mathrm{ml}$ in phosphate-buffered saline (PBS), and shipped to the New York State Agricultural Experiment Station, Geneva. C. parvum oocysts were suspended in 2 liters of fresh apple cider prepared at the New York State Agricultural Experiment Station to a final concentration of $1 \times 10^{5}$ to $10 \times 10^{5}$ oocysts $/ \mathrm{ml}$. There are no industry standards for fresh cider; however, fresh apple cider is commonly defined as juice expressed from whole apples that may or may not be filtered to remove solids and has a relatively short refrigerated shelf life. The entire 2 liters of inoculated apple cider were then subjected to $14.32 \mathrm{~mJ}$ of UV irradiation/ $/ \mathrm{cm}^{2}$ for 1.2 to $1.9 \mathrm{~s}$ by a CiderSure $3500 \mathrm{~A}$ apparatus (FPE Inc., Rochester, N.Y.). This UV apparatus is comprised of a stainless steel outer housing and an inner quartz tube. The cider is passed between the outer steel housing and inner quartz tube during UV treatment. Eight germicidal low-pressure mercury lamps are placed concentrically within the interior of the quartz-stainless steel cylinder to provide UV light exposure to the passing fluid. Two UV light sensors are placed at the top and bottom of the cylinder. UV sensor readings are then taken every $50 \mathrm{~ms}$, and the values are directly relayed to the control panel. A calculated algorithm for the control panel which achieves a $5 \log _{10}$ reduction of $E$. coli $\mathrm{O} 157: \mathrm{H} 7$ in unfiltered apple cider with the UV apparatus is then used. The apparatus takes into consideration the differences in apples cider qualities, i.e., solids and color, through the UV sensor 


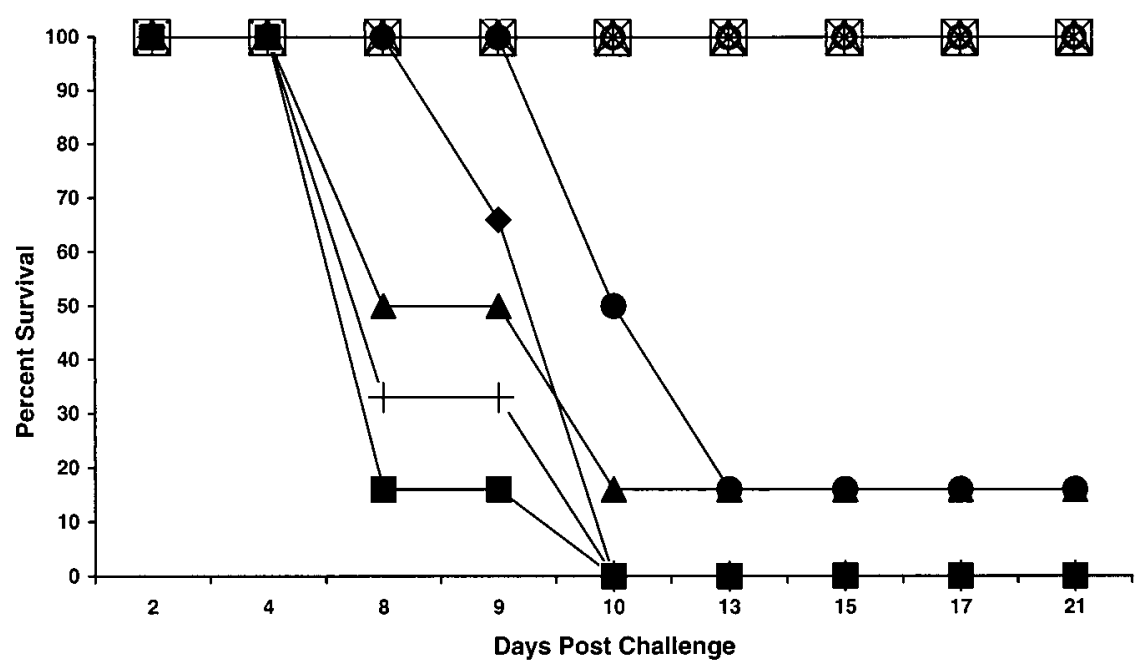

FIG. 1. GKO mouse survival after challenge with UV-irradiated apple cider or untreated apple cider inoculated with C. parvum oocysts. Groups of six animals each were challenged by oral gavage with UV-irradiated apple cider containing $10^{3}$ oocysts $(\triangle), 10^{4}$ oocysts $(\diamond), 10^{5}$ oocysts $(\square)$, or $10^{6}$ oocysts $(\bigcirc)$ or with untreated cider containing $10^{3}$ oocysts $(\bullet), 10^{4}$ oocysts $(\bullet), 10^{5}$ oocysts $(\square)$, or $10^{6}$ oocysts $(\mathbf{\Delta})$. Control groups were challenged with uninoculated cider or $10^{5}$ oocysts in PBS $(+)$.

data acquisition. UV exposure readings automatically change the pump flow rate to ensure a $14.32-\mathrm{mJ} / \mathrm{cm}^{2}$ exposure level throughout the entire sample. Passage of cider through the apparatus results in a temperature increase of only $0.1^{\circ} \mathrm{F}$ throughout the unit. The temperature of the cider when the oocysts were added and following passage through the UV apparatus remained a constant $38^{\circ} \mathrm{F}$. A second 2-liter aliquot of the same lot of apple cider was inoculated with $1 \times 10^{5}$ to $10 \times 10^{5}$ oocysts/ml and used for the non-UV-treated portion of the study. An additional portion of cider from the same production lot was used as an uninoculated control and diluent. Samples were shipped to the Food and Drug Administration (FDA) laboratories in Laurel, Md., and the Centers for Disease Control and Prevention laboratories in Atlanta, Ga., for viability testing. The UV-treated and untreated apple cider samples inoculated with oocysts were 10 -fold serially diluted in cider to final concentrations of $2 \times 10^{3}$ oocysts per ml. As necessary, oocyst suspensions were concentrated by centrifugation to achieve inoculum volumes of 25 to $100 \mu \mathrm{l}$ for the neonatal BALB/cByJ mouse pup studies. Flow cytometry was used to verify the presence of oocysts in all cider preparations.

Oocyst viability was determined with the gamma interferon knockout mouse model following the methods of Griffiths et al. (12) and Theodos et al. (21). All studies were conducted in compliance with the Guide for the Care and Use of Laboratory Animals (17). Female 8-week-old C57BL/6 gamma interferon gene knockout (GKO) mice (Jackson Laboratories, Bar Harbor, Maine) were randomized into challenge groups of six mice each and control groups of three mice each. Mice were challenged by oral gavage with a sterile 22-gauge feeding needle attached to a 3-ml disposable syringe. Animals in four of the challenge groups received untreated apple cider containing oocysts at concentrations of $10^{6}, 10^{5}, 10^{4}$, and $10^{3}$ oocysts per animal, while animals in the other four groups received UVirradiated apple cider containing oocysts at concentrations of $10^{6}, 10^{5}, 10^{4}$, and $10^{3}$ oocysts per animal. Three mice were each challenged with $10^{5}$ oocysts suspended in PBS as a positive control to confirm the viability of oocysts following shipment. An additional three mice were challenged with the diluent apple cider as negative controls. Animals were observed on a daily basis for morbidity and mortality. Fecal pellets were collected from each animal before challenge and then weekly postchallenge to monitor oocyst shedding. Fecal pellets were homogenized in $2.5 \%$ potassium dichromate and assayed for C. parvum by a PCR assay as previously described $(7,14,19)$. GKO mice that survived 21 days postchallenge were euthanized by the Euthanex system (Euthanex Corp., Bethlehem, Pa.). The mid- to posterior portion of the small intestine (jejunum to ileum) was removed from all mice, and tissues were paraffin embedded, thin sectioned, stained with hematoxylin and eosin stain, and evaluated by light microscopy. Intestinal tissues in 1- to $2-\mathrm{cm}^{2}$ sections were minced and scraped in buffer with a sterile scalpel, homogenized, and assayed for oocysts by following the PCR methods described above.

All adult GKO mice challenged with UV-treated apple cider containing from $10^{3}$ to $10^{6}$ oocysts showed no clinical signs of disease and survived for the course of the study (Fig. 1). Necropsy results showed no marked changes in the intestinal tracts of these animals when compared to those of untreated control animals, and histology results revealed normal intestinal architecture and no indications of a parasitic infection. PCR was used to detect the presence of oocysts in the feces, which is indicative of an active infection with C. parvum. Figure $2 \mathrm{~A}$ shows the expected PCR product when oocysts are used in the assay. Oocysts from both UV-treated and untreated cider were used in the assay to demonstrate that UV treatment did not interfere with PCR results. PCR (Fig. 2B) analyses of fecal specimens were negative for the presence of $C$. parvum for the course of the study, showing that these mice were not infected with the parasite. In addition, results from the PCR assay of tissue sections from animals challenged with $10^{6}$ oocysts in UV-treated cider were also negative for C. parvum (Fig. 2C). 
$\mathbf{A}$

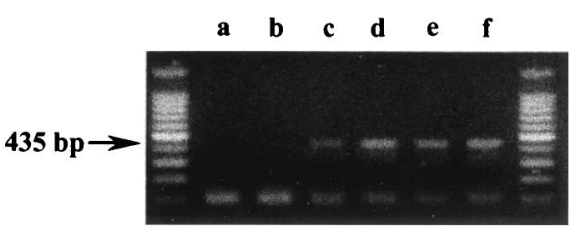

Untreated

B

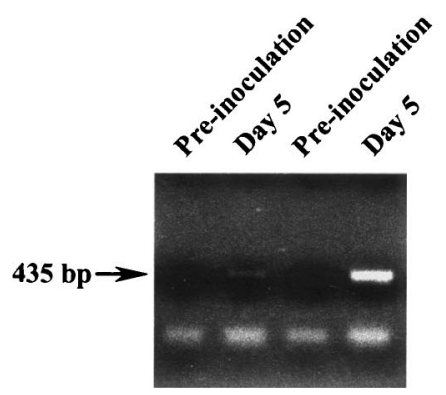

Inoculum: $10^{3} \quad 10^{3} \quad 10^{6} \quad 10^{6}$

Untreated

C

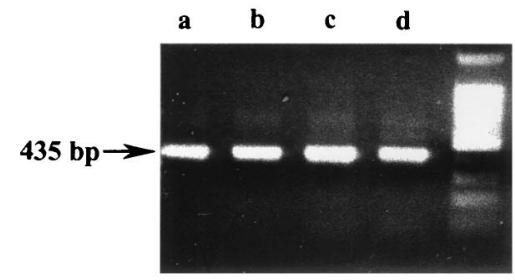

Inoculum: $\mathbf{1 0}^{3}$

Untreated

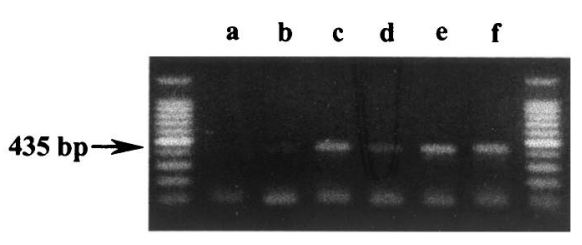

UV-irradiated
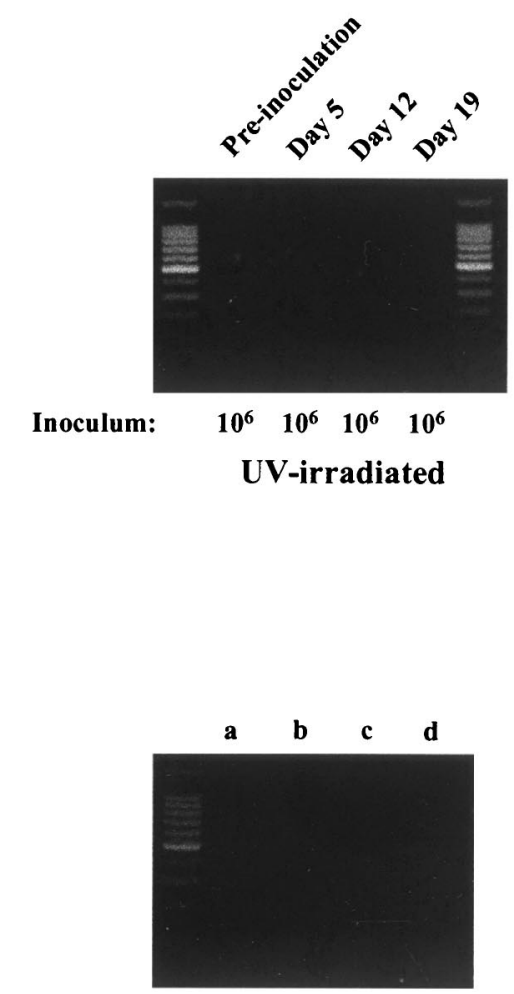

Inoculum: $10^{6}$

\section{UV-irradiated}

FIG. 2. Apple cider inoculums used as PCR controls, fecal specimens, and intestinal tissue were analyzed by PCR for the presence or absence of $C$. parvum. (A) Aliquots of untreated and UV-irradiated apple cider containing $10^{5}$ C. parvum oocysts $/$ ml were spotted directly onto fluorescent treponemal antibody (FTA) filter disks and analyzed by PCR. Lanes a through f represent 1-, 3-, 5-, 10-, and 20- $\mu 1$ samples, respectively. (B) Fecal specimens from GKO mice inoculated at the indicated dosage with either untreated or UV-irradiated C. parvum-containing apple cider were analyzed by PCR for the presence of $C$. parvum. (C) Intestinal tissue isolated from GKO mice inoculated with either untreated $C$. parvumcontaining apple cider $\left(10^{3}\right.$ oocysts) or UV-irradiated C. parvum-containing apple cider $\left(10^{6}\right)$ was analyzed by PCR for the presence of $C$. parvum. Lanes a through d represent 1, 5, 10, and $20 \mu \mathrm{l}$ of tissue homogenate applied to FTA filter disks, respectively. Unmarked lanes contain 100-bp standards.

In contrast, mice challenged with oocyst-inoculated cider that was not UV treated demonstrated the marked clinical symptoms of infection, including weight loss and lethargy, and died within 13 days postchallenge (Fig. 1). There was no significant difference in outcome between animals challenged with oocysts suspended in PBS and those challenged with oocysts suspended in apple cider (Fig. 1), indicating that exposure to apple cider alone does not affect the virulence of $C$. parvum oocysts. Fecal pellets collected from all animals challenged with untreated inoculated apple cider, including the two mice that survived challenge, or with oocysts suspended in PBS were positive for $C$. parvum based on enzyme-linked immunosorbent and PCR assays. As shown in Fig. 2B, oocysts could be detected in feces from mice in both the high- and low-dose groups by 5 days postinfection. Oocysts were not detected in animals prior to challenge (Fig. 2B). This further demonstrates an active infection with $C$. parvum initiated by challenge with non-UV-treated apple cider containing viable $C$. parvum oo- 
cysts. Necropsy results revealed a distended, dilated intestinal tract indicative of infection in all animals challenged with nonUV-treated inoculated cider, and histopathology results revealed Cryptosporidium in various stages of development in the enterocytes near the tips of the microvilli. PCR analysis of intestinal tissue from the single mouse that survived challenge with $10^{6}$ oocysts confirmed the presence of C. parvum in this animal (Fig. 2C). Animals challenged with cider alone had no clinical signs of illness for the duration of the study, showing that cider alone does not cause illness in GKO mice.

Studies were also conducted in parallel with neonatal $\mathrm{BALB} / \mathrm{cByJ}$ mice to corroborate data from the GKO studies. Female, timed-pregnant BALB/cByJ inbred mice were obtained from Jackson Laboratories. Pups approximately 5 to 8 days old were randomized into challenge groups of four to eight animals each and control groups containing three to four animals each. Four groups of pups received UV-treated apple cider $\left(50 \mu \mathrm{l}\right.$ each) containing oocysts at concentrations of $10^{6}$, $10^{5}, 10^{4}$, and $10^{3}$ oocysts per animal. Mice in two control groups received untreated apple cider containing oocysts at concentrations of $10^{4}$ and $10^{3}$ oocysts per animal. The negative control group received uninoculated apple cider. A final group of five mice was challenged with $10^{3}$ oocysts per mouse in PBS to verify that shipping conditions were not responsible for oocyst inactivation independent of UV treatment. All pups were challenged by oral gavage. Inoculated mice were maintained approximately 1 week before evaluating evidence of infection to allow for small numbers of infectious oocysts to establish a detectable infection in mice. Neonatal mice were humanely euthanized by $\mathrm{CO}_{2}$ inhalation, and the terminal colon below the cecum was removed from each animal and placed in a siliconized microcentrifuge vial containing $400 \mu \mathrm{l}$ of $2.5 \%$ potassium dichromate. A wooden applicator was used to express the intestinal contents and then remove the segment of colon. The intestinal contents were homogenized into the potassium dichromate with the same wooden applicator and extensive vortexing. The homogenates were subjected to a microscale variation of the discontinuous sucrose gradient method described for the purification of oocysts from calf stool $(1 \mathrm{a}, 2)$. The gradient interface was collected, washed by centrifugation $(16,000 \times g)$ with saline, and suspended in $300 \mu \mathrm{l}$ of PBS (0.01 M, pH 7.2) supplemented with $0.1 \%$ bovine serum albumin. The partially purified concentrate was incubated for $30 \mathrm{~min}$ at $37^{\circ} \mathrm{C}$ with $5 \mu \mathrm{l}$ of an oocyst-specific monoclonal antibody conjugated with fluorescein isothiocyanate (OW50FITC) at a 1/50 dilution in PBS. Samples were adjusted to 600 $\mu l$ with PBS and analyzed by flow cytometry with a FACScan (Becton Dickinson, Mountainview, Calif.). Samples were evaluated by using a 102-s sampling interval $(100 \mu \mathrm{l})$, with logical gating of the forward- and sidescatters and OW50-FITC fluorescence signals on the flow cytometer (1). Data files were collected by a flow cytometer and subsequently analyzed using software provided with the FACScan (Lysis II; Becton Dickinson). Each experimental run included positive and negative controls used to calibrate the region settings necessary to discriminate between the labeled oocyst population and background debris. Absolute counts were calculated from the data files for oocysts per $100 \mu \mathrm{l}$ of sample suspension. Mouse infection data were analyzed as previously described $(11,24)$.

Infections were not seen in any neonatal BALB/c mice chal-
TABLE 1 . UV inactivation of $C$. parvum oocysts assayed in a neonatal $\mathrm{BALB} / \mathrm{c}$ mouse model

\begin{tabular}{|c|c|c|c|c|c|}
\hline \multirow[t]{2}{*}{ Treatment $^{a}$} & \multirow{2}{*}{$\begin{array}{c}\text { No. of } \\
\text { oocysts/dose/mouse }\end{array}$} & \multirow[t]{2}{*}{$n$} & \multirow{2}{*}{$\begin{array}{c}\% \\
\text { Infected }\end{array}$} & \multicolumn{2}{|c|}{$\begin{array}{l}\log _{10} \\
\text { reduction }\end{array}$} \\
\hline & & & & Minimum $^{b}$ & Maximum $^{c}$ \\
\hline Cider control & 0 & 4 & 0 & 0.00 & 0.00 \\
\hline Trip control & $10^{3}$ & 5 & 100 & 0.64 & 0.64 \\
\hline Untreated & $10^{3}$ & 5 & 100 & 0.64 & 0.64 \\
\hline Untreated & $10^{4}$ & 4 & 100 & 0.64 & 0.64 \\
\hline UV & $10^{3}$ & 5 & 0 & 1.66 & 2.99 \\
\hline UV & $10^{4}$ & 5 & 0 & 2.66 & 4.00 \\
\hline UV & $10^{5}$ & 7 & 0 & 3.66 & 5.00 \\
\hline UV & $10^{6}$ & 12 & 0 & 4.66 & 6.00 \\
\hline
\end{tabular}

${ }^{a}$ Trip control oocysts included to verify shipping conditions between institutions did not influence oocyst infectivity. The control consisted of untreated apple cider supplemented with oocysts at $10^{6} / \mathrm{ml}$ and then diluted to the appropriate dose per mouse. UV treatment was applied to apple cider supplemented with oocysts at $10^{6} / \mathrm{ml}$ and then diluted to the appropriate dose per mouse.

${ }^{b}$ Minimum infectivity reduction based on the oocyst detection limit of 23 oocysts $(11,24)$.

$c$ Maximum infectivity reduction assuming no infective oocysts remained in the sample.

lenged with UV-treated apple cider containing from $10^{3}$ to $10^{6}$ oocysts (Table 1). There was no difference between infectivity rates of animals receiving oocysts in PBS and those receiving oocysts in cider, indicating that cider does not affect oocyst viability. In contrast, flow cytometric analysis showed that all neonatal $\mathrm{BALB} / \mathrm{c}$ mice inoculated with untreated inoculated cider became infected while mice receiving apple cider alone remained uninfected (Table 1).

This study evaluated the effectiveness of UV irradiation in the inactivation of $C$. parvum oocysts in fresh apple cider. Two established animal models, the GKO mouse and the neonatal mouse, were used to determine the viability of $C$. parvum oocysts. Dose-response data from previous studies done in our laboratory show that the GKO mouse is sensitive to $\leq 10$ viable oocysts when death is used as the endpoint. When pathological changes are used as the criteria for infection, GKO mice were sensitive to one oocyst. In the neonatal mouse model, the minimum detection limit was 23 oocysts. The sensitivity of these models allowed for the detection of low numbers of viable oocysts that may have been present in UV-treated samples.

A maximum concentration of $10^{6}$ oocysts $/ \mathrm{ml}$ of cider used for these experiments most likely represents an extreme level of contamination; however, few data are available on the concentration of $C$. parvum in fresh cider. These numbers were also required to determine the optimum $\log _{10}$ kill that could be achieved through UV irradiation. To meet FDA guidelines for fresh juices (23), a postproduction process must achieve a 5 $\log _{10}$ reduction in pathogen load in the finished product. Results from the GKO mouse show a maximum $6 \log _{10}$ reduction and a minimum $5 \log _{10}$ reduction that is well within the FDA guidelines. In this study, one GKO mouse challenged with $10^{6}$ UV-untreated oocysts survived the 21-day study. However, PCR of fecal samples and intestinal tissue were positive for $C$. parvum at day 21 postinfection, indicating an active infection. Biological differences among animals often result in responses that are different from those expected. Problems with oral dosing that occasionally result in the mouse not receiving the 
entire inoculum may also account for variable results. Data obtained from the neonatal BALB/c model were consistent with those obtained from the GKO model. A maximum $6 \log _{10}$ and a minimum $4.66 \log _{10}$ reduction were obtained in these animals.

In conclusion, data generated from both the gamma interferon GKO mouse and the neonatal BALB/c mouse models show that exposure to $14.32 \mathrm{~mJ}$ of UV irradiation $/ \mathrm{cm}^{2}$ for $\leq 1.9$ s can achieve the requirement of a $5 \log _{10}$ reduction in viable C. parvum present in apple cider. Data from these studies further suggests that a greater than $5 \log _{10}$ reduction may be possible by using the CiderSure apparatus and the tested UV parameters. These pathogen reduction values meet the $5 \log _{10}$ criterion for postproduction processing. Therefore, UV treatment of apple cider is a feasible technology for the reduction of viable $C$. parvum oocysts in fresh apple cider.

We thank Colleen Carroll for critical reading and editing of the manuscript.

\section{REFERENCES}

1. Arrowood, M. J., M. R. Hurd, and J. R. Mead. 1995. A new method for evaluating experimental cryptosporidial parasite loads using immunofluorescent flow cytometry. J. Parasitol. 81:404-409.

1a.Arrowood, M. J., and K. Donaldson. 1996. Improved purification methods for calf-derived Cryptosporidium parvum oocysts using discontinuous sucrose and cesium chloride gradients. J. Eukaryot. Microbiol. 43(Suppl.):89.

2. Arrowood, M. J., and C. R. Sterling. 1987. Isolation of Cryptosporidium oocysts and sporozoites using discontinuous sucrose and isopycnic Percoll gradients. J. Parasitol. 73:314-319.

3. Bachman, R. 1975. Sterilization by intense ultraviolet radiation. Brown Boveri Rev. 62:206-209.

4. Centers for Disease Control and Prevention. 1997. Outbreaks of Escherichia coli infection and cryptosporidiosis associated with drinking unpasteurized apple cider-Connecticut and New York, October 1996. Morb. Mortal. Wkly. Rep. 46:4-8.

5. Craik, S. A., D. Weldon, G. R. Finch, J. R. Bolton, and M. Belosevic. 2001. Inactivation of Cryptosporidium parvum oocysts using medium- and lowpressure ultraviolet radiation. Water Res. 35:1387-1398.

6. Crawford, F. G., and S. H. Vermund. 1998. Human cryptosporidiosis. Crit. Rev. Microbiol. 16:113-159.

7. De Boer, S. H., L. J. Ward, X. Li, and S. Chittaranjan. 1995. Attenuation of PCR inhibition in the presence of plant compounds by addition of BLOTTO. Nucleic Acids Res. 23:2567-2568.

8. Downing, D. L. (ed.). 1989. Processed apple products. Van Nostrand Reinhold, New York, N.Y.
9. Fayer, R., C. A. Speer, and J. P. Dubey (ed.). 1990. General biology of Cryptosporidium, p. 1-29. In Cryptosporidiosis of man and animals. CRC Press, Boca Raton, Fla.

10. Fayer, R., and B. L. P Ungar. 1986. Cryptosporidium spp. and cryptosporidiosis. Microbiol. Rev. 50:458-483.

11. Finch, G. R., C. W. Daniels, E. K. Black, F. W. Schauder, and M. Belosevic. 1993. Dose response of Cryptosporidium parvum in outbred neonatal CD-1 mice. Appl. Environ. Microbiol. 59:3661-3665.

12. Griffiths, J. K., C. Theodos, M. Paris, and S. Tzipori. 1998. The gamma interferon gene knockout mouse: a highly sensitive model for evaluation of therapeutic agents against Cryptosporidium parvum. J. Clin. Microbiol. 36: 2503-2508.

13. Harrington, W. O., and C. H. Hills. 1968. Reduction of the microbial population of apple cider by ultraviolet irradiation. Food Technol. 22:117-120.

14. Johnson, D. W., N. J. Pieniazek, D. W. Griffin, L. Misener, and J. B. Rose. 1995. Development of a PCR protocol for the sensitive detection of Cryptosporidium oocysts in water samples. Appl. Environ. Microbiol. 61:38493855.

15. Laberge, I., and M. W. Griffiths. 1996. Prevalence, detection and control of Cryptosporidium parvum in food. Int. J. Food Microbiol. 32:1-26.

16. Millard, P. S., K. F. Gensheimer, D. G. Addise, D. M. Sosin, G. A. Beckett, A. Houck-Jankowski, and A. Hudson. 1994. An outbreak of cryptosporidiosis from fresh-pressed apple cider. JAMA 272:1592-1596.

17. National Research Council. 1996. Guide for the care and use of laboratory animals. National Academy Press, Washington, D.C.

18. O'Donoghue, P. J. 1995. Cryptosporidium and cryptosporidiosis in man and animals. Int. J. Parasitol. 25:139-195.

19. Orlandi, P. A., and K. A. Lampel. 2000. Extraction-free, filter-based template preparation for rapid and sensitive PCR detection of pathogenic parasitic protozoa. J. Clin. Microbiol. 38:2271-2277.

20. Siobain, D., J. Churey, R. W. Worobo, and D. W. Schaffner. 2000. Analysis and modeling of the variability associated with UV inactivation of Escherichia coli in apple cider. J. Food Protect. 63:1587-1590.

21. Theodos, C. M., K. L. Sullivan, J. K. Griffiths, and S. Tzipori. 1997. Profiles of healing and nonhealing Cryptosporidium parvum infection in $\mathrm{C} 57 \mathrm{Bl} / 6$ mice with functional B and T lymphocytes: the extent of gamma interferon modulation determines the outcome of infection. Infect. Immun. 65:4761-4769.

22. Ungar, B. L. P. 1990. Cryptosporidiosis in humans (Homo sapiens), p. 59-82. In J. P. Dubey, C. A. Speer, and R. Fayer (ed.), Cryptosporidiosis of man and animals. CRC Press, Boca Raton, Fla.

23. U.S. Food and Drug Administration. 1998. Hazard analysis and critical control point (HACCP): procedures for the safe and sanitary processing and importing of juice, 21 CFR, part 120. Fed. Regist. 63:20449-20486.

24. Venczel, L. V., M. Arrowood, M. Hurd, and M. D. Sobsey. 1997. Inactivation of Cryptosporidium parvum oocysts and Clostridium perfringens spores by mixed-oxidant disinfectant and by free chlorine. Appl. Environ. Microbiol. 63:1598-1601.

25. Wright, J. R., S. S. Sumner, C. R. Hackney, M. D. Pierson, and B. W. Zoecklein. 2000. Efficacy of ultraviolet light for reducing Escherichia coli O157:H7 in unpasteurized apple cider. J. Food Protect. 63:563-567.

26. Yip, R. W., and D. E. Konasewich. 1972. Ultraviolet sterilization of waterits potential and limitations. Water Pollut. Control 1972:14-18. 